\title{
ON A DOUBLE EIGENVALUE PROBLEM
}

\section{A. D. ZIEBUR}

In the study of the rigidity of surfaces of revolution one encounters the following "double" eigenvalue problem $[2 ; 3]$ : Find those numbers $a>0$ for which there exists a not identically vanishing function $y$ and an integer $m>1$ such that $y$ is a solution of the system

$$
\left(\frac{y^{\prime}}{p(x)}\right)^{\prime}+\lambda \frac{q(x)}{(a+r(x)) p^{2}(x)} y=0, \quad y(0)=y(b)=0,
$$

where $\lambda=m^{2}-1$. Rembs [3] demonstrated the existence of a countably infinite set of such $a$ 's under the assumption that the functions $p, q$, and $r$ are analytic on the interval $[0, b]$, that $q>0$ and $r \geqq 0$ on the interval, and that $p>0$ on the open interval, but vanishes to the first order at each end. Minagawa and Rado, in a more general setting [2], showed that these "exceptional $a$ 's" are at most countable in number. It is the purpose of the present note to show that the $a$ 's form a dense set on the positive real axis. For the validity of the following argument it is sufficient to assume that the functions $p, q$, and $r$ are of class $C^{\prime \prime}$ rather than analytic and that $p$ vanishes, if at all, to a lower order than the second at the end points.

For a fixed positive number $a$, the problem (1) is a (regular singular) Sturm-Liouville eigenvalue problem. It is known that the eigenvalues $\lambda_{n}(a)$ are positive and can be arranged in the sequence $0<\lambda_{1}(a)$ $<\lambda_{2}(a)<\cdots \rightarrow \infty$. Further, each $\lambda_{n}(a)$ is a continuous function of $a$ on any interval $0<a_{1} \leqq a \leqq a_{2}$. Our problem, then, is to study those values of $a$ for which at least one member of the sequence of eigenvalues has the form $\lambda_{n}(a)=m^{2}-1$. Now let $R=\max r(x)$ and rewrite problem (1),

$$
\left(\frac{y^{\prime}}{p}\right)^{\prime}+\omega \frac{q}{(1-(R-r) /(a+R)) p^{2}} y=0, \quad y(0)=y(b)=0,
$$

where $\omega=\lambda /(a+R)$. The $n$th eigenvalue of problem (2) is therefore given by the formula $\omega_{n}(a)=\lambda_{n}(a) /(a+R)$. Now the coefficient of $\omega y$ is a decreasing function of $a$. Hence, if $a_{1}<a_{2}$, a known theorem [ 1 , p. 357] tells us that $\omega_{n}\left(a_{1}\right) \leqq \omega_{n}\left(a_{2}\right)$, i.e.,

$$
\frac{\lambda_{n}\left(a_{1}\right)}{a_{1}+R} \leqq \frac{\lambda_{n}\left(a_{2}\right)}{a_{2}+R} \text {. }
$$

Presented to the Society, September 3, 1953; received by the editors June 10, 1953 and, in revised form, July 16, 1953. 
Finally, let us note that, for each $a, \lim _{n} \lambda_{n}(a) n^{-2}$ exists and is positive $[1$, p. 361$]$. It therefore follows that

$$
\lim _{n \rightarrow \infty} \frac{\lambda_{n}(a)}{\lambda_{n+1}(a)}=1 .
$$

We are now in a position to prove our main result.

THEOREM. Let $a_{1}$ and $a_{2}$ be any two given numbers such that $0<a_{1}<a_{2}$. Then there exists an integer $m>1$ and $a$ number $a$, where $a_{1} \leqq a \leqq a_{2}$, such that $\lambda_{n}(a)=m^{2}-1$ for some $n$.

Proof. Since $a_{1}<a_{2}$, we have $\left(a_{1}+R\right) /\left(a_{2}+R\right)<1$. As a result of (4) there exists an integer $N$ such that for all $n \geqq N$

$$
\frac{\lambda_{n}\left(a_{1}\right)}{\lambda_{n+1}\left(a_{1}\right)}>\frac{\left(a_{1}+R\right)}{\left(a_{2}+R\right)} .
$$

Now pick $m$ so that $m^{2}-1 \geqq \lambda_{N}\left(a_{1}\right)$ and let $n(\geqq N)$ be the number such that $\lambda_{n}\left(a_{1}\right) \leqq m^{2}-1<\lambda_{n+1}\left(a_{1}\right)$. From the inequalities (3) and (5) we can conclude that $\lambda_{n+1}\left(a_{1}\right) \leqq \lambda_{n}\left(a_{2}\right)$ so that $\lambda_{n}\left(a_{1}\right) \leqq m^{2}-1<\lambda_{n}\left(a_{2}\right)$. But $\lambda_{n}(a)$ is a continuous function of $a$ on the interval $\left[a_{1}, a_{2}\right]$, so there must be a value of $a$ in that interval for which $\lambda_{n}(a)=m^{2}-1$.

\section{BIBLIOGRAPHY}

1. R. Courant and D. Hilbert, Methoden der mathematischen Physik, vol. 1, 2d ed., Berlin, 1931.

2. T. Minagawa and T. Rado, On the infinitesimal rigidity of surfaces of revolution, Math. Zeit. vol. 59 (1953) pp. 151-163.

3. E. Rembs, Über die Verbiegung parabolisch berandeter Flächen negativer Krümmung, Math. Zeit. vol. 35 (1932) pp. 529-535.

The Ohio State University 